\title{
GROWING UP PAINS IN A DEVELOPING COUNTRY
}

\author{
S. TORRES-PEIMBERT \\ Instituto de Astronomía, Universidad Nacional Autónoma de México \\ Ap. Postal 70-264, México 04510, D.F., México, \\ e-mail: silvia@astroscu.unam.mx
}

\section{Introduction}

Similarly to other developing nations, Mexico is a country full of contrasts and contradictions; within the country there are many very backward conditions, and yet it strives to participate in advanced scientific activities.

The country has 95 million inhabitants, the median age of the population is 18 years, the fraction of teenagers that attend upper high school is $30 \%$ and the fraction of those in the 18-22 age bracket that attend college is $14 \%$. Finally it should be pointed out that the expenditure in science amounts to $0.3 \%$ of the Gross National Product, GNP. These figures should be compared with developed countries, where $85 \%$ of the youngsters attend high school, $45 \%$ of those in the 18-22 age bracket attend college, and typically $1 \%$ of the GNP is spent on science.

The situation in astronomy is varied in different regions of the country. There are universities with well established scientific groups, but in many others there is hardly any interest in research in any subject. Scientific research is carried out in public universities, while private universities have not considered it at all.

\section{Present conditions of astronomy}

For the last 35 years, the largest institution has been the Instituto de Astronomia of Universidad Nacional Autónoma de México, IAUNAM. It has grown from 10 astronomers in 1965 to 75 in 1997. The number of technical support staff has grown in a similar fashion. IAUNAM has 3 research locations: one in the main campus in Mexico City, another one in Ensenada, Baja California and a third one in Morelia, Michoacan. In addition there are two observing sites that will be described below.

Another important institution is the Instituto Nacional de Astrofísica, Optica y Electrónica, INAOE, located at Tonantzintla, Puebla; it has increased its effort in astronomy in recent years and now has 22 astronomers. A new astronomy department has been started at the Universidad de Guanajuato, in Guanajuato City, which now has 8 astronomers. In addition, there are several astronomers in physics departments in different universities in the country. Altogether the total number of professional astronomers in Mexico is of 110 .

\section{Observing facilities}

The main observing site of the IAUNAM, is in San Pedro Mártir, Baja California with 3 telescopes: $2.1-\mathrm{m}, 1.5-\mathrm{m}$ and $0.84-\mathrm{m}$. The instrumentation available is direct image, spectroscopy and photometry and near-infrared direct image and spectroscopy. In addition there is a 1-m telescope in Tonantzintla, Puebla, with options for direct inage, spectroscopy and photometry, although the atmosphoric conditions of the site are rather poor.

The observing site of INAOE, is at Cananea, Sonora (very close to the MTT in Arizona), where there is a $2.1-\mathrm{m}$ telescope with a multiobject spectrograph (in collaboration with University of Heidelberg), and the 27/31-in Schmidt camera in Tonantzintla, Pucbla, where the observing conditions are not favorable. 


\subsection{FUTURE TELESCOPES}

INAOE is constructing, a 50-m radio antenna for millimeter wavelengths to be built on Cerro de la Negra in Puebla. This project is in collaboration with the University of Massachusetts.

IAUNAM has the project of building a 6.5-m segmented optical/near-ir telescope in San Pedro Mártir, Baja California. The design is modular and is based on an array of 19 hexagons each of $1.8-\mathrm{m}$. At present this project has not been fully funded, but the first phase of construction is expected to begin soon.

\section{Graduate programs}

IAUNAM based the education of astronomers on sending students to graduate school in the US and Europe, at first because there were not enough trained astronomers for that activity, and later it was thought desirable to diversify topics and schools of thought. Only gradually a graduate program was started at IAUNAM based on the physics program which later developed into a full fledged astronomy program. 11 Master's and $9 \mathrm{PhD}$ degrees have been granted in the physics program to students that were trained in astronomy; and 23 Master's and $4 \mathrm{PhD}$ degrees have been granted in the astronomy program that was started in 1989. At the same time, since 1990 the INAOE started its own graduate program from which there have been 6 Master's and $1 \mathrm{PhD}$ degrees granted.

The programs are successful and from the present enrollment it is clear that more students are expected to obtain their degrees in astronomy in the near future. However, there are several problems: (1) There is a need to strengthen research in the smaller universities, and that includes developing astronomy departments or groups of astronomers in physics departments. However the students who obtain their degrees do not want to spend all their effort establishing a new department, when the funding agencies require results measured in number of 'research papers'. (2) An additional problem is that the number of scholarships available is shrinking which can limit severely the number of students enrolled in graduate programs. (3) At present most of the observational astronomers in Mexico require optical telescopes and the resources in that area have not increased in step with the number of astronomers. Thus we require significant improvements on the optical (and infrared) observing facilities; in particular I consider that we require a stronger commitment from the funding agencies for a large optical/ir telescope.

\section{Conclusions}

There are two graduate programs in Astronomy in Mexico, one at the IAUNAM and another one at the INAOE, that although they have started fairly recentiy they are working out successfully.

However, we note that there are about 110 professional astronomers in the country, which corresponds to 1 astronomer per million inhabitants; while in developed countries there are from 20 to 30 astronomers per million inhabitants. That is, the country needs to prepare the students and to create the positions for the most gifted ones to follow a research career. The conclusion is clear for us: WE NEED TO WORK HARDER! 